*Proceedings*

\title{
Self-assembly and Electrorheological Effect of Phthalocyanine-Based Liquid Crystalline Polysiloxanes ${ }^{\dagger}$
}

\author{
Pingwei Sun, Fanbao Meng * and Xinqiao Tang \\ College of Science, Northeastern University, Shenyang 110819, China; email1@gmail.com (P.S.); \\ email2@gmail.com (X.T.) \\ * Correspondence: mengfb @ mail.neu.edu.cn; Tel.: +86-024-8368-7671 \\ + Presented at the First International Conference on “Green" Polymer Materials 2020, 5-25 November 2020; \\ Available online: https://cgpm2020.sciforum.net/.
}

Published: 3 November 2020

\begin{abstract}
A series of phthalocyanine-based liquid crystalline polysiloxanes (PLCPs) were synthesized by use of poly(methylhydrogeno)siloxane (polymer matrix), a sulfonic acid-containg monomer 4-(allyloxy)benzenesulfonic acid, a liquid crystal monomer cholesteryl 4(allyloxy)benzoate and a phthalocyanine-containing monomer zinc tetraaminophthalocyanine. The chemical structure, liquid crystal, dielectric and electrorheological properties were characterized via various experimental techniques. With increase of phthalocyanine in the supermolecular systems, the mesophases of PLCPs change from nematic phase to discotic hexagonal columnar mesophase. Furthermore, the dielectric constant increases with increase of phthalocyanine component in the polymer systems. All the PLCPs exhibit electrorheological (ER) effect. For the ER fluid of PLCPs prepared by the same method, the ER effect strengthens firstly and then weakens with increase of phthalocyanine component in the polymers. The ER effect is strongest when the molar ratio of phthalocyanine and cholesteryl mesogens in the polymer is 8:2. This suggests some synergistic effect is occurred between semiconducting property and molecular orientation in these phthalocyaninebased liquid crystalline polysiloxanes.
\end{abstract}

Keywords: self-assembly; electrorheological; phthalocyanine; liquid crystal

\section{Introduction}

As a kind of smart materials, electrorheological fluids (ERFs) can reversibly and rapidly change electrorheology and structure exhibiting a tremendous viscosity change from a liquid- to a solid-like state as applied by an electric field strength, and the ERFs have attracted lasting interest in material science [1]. Typical ERFs are suspensions of polarizable or conducting particles dispersing in nonpolar oil, and the ER effect is due to formation of a chain-like structure of particles along the direction of the applied electric field strength. Lots of particles have been used as ER materials including conducting polymers and high dielectric inorganics as well as their composites [2]. However, in spite of the broad interest, suspension ERFs still possess some major disadvantages including particle attrition and settling, especially over prolonged times. In an effort to overcome these disadvantages, some ER materials based on poly(ionic liquid) or liquid crystal have been studied [3]. Liquid crystal materials have been observed a variety of ER effects and used as ER materials depending on the characteristic structures of the respective liquid crystalline phases [4]. Ionic liquid crystal is a selforganizing system combining both ionic liquid and liquid crystal characteristics and shows additional performance such as ionic conductivity, self-assembling ability, anisotropic physical properties, dynamic molecular order, tuning possibilities, and so on. In this work, ionic PLCPs were 
synthesized and applied as ER materials. The effect of the PLCP's structure on the performances including liquid crystalline behavior, polarization property and ER effect was investigated in this study.

\section{Experiments}

\subsection{Materials and Techniques}

poly(methylhydrogen)siloxane $\left(M_{\mathrm{n}} \approx 2300\right)$, 3-bromopropane, cholesterol, 4-hydroxybenzoic acid, 4-hydroxybenzenesulfonic acid, zinc tetranitrophthalocyanine, silicone oil (polydimethylsiloxane, viscosity $500 \mathrm{cSt}$, dielectric constant 2.6-2.7) and sodium sulfide were obtained from Alfa Aisha (China) Chemical Co. Ltd. and used without any further purification. Other chemical reagents were purchased from Beijing HWRK Chemical Company.

Fourier-transform infrared (FTIR) spectra were recorded on PerkinElmer instruments Spectrum One Spectrometer (PerkinElmer, Foster City, CA) by the potassium bromide method. The element analyses were performed with an Elementar Vario EL III (Elementar, Germany). Thermal properties were determined by a differential scanning calorimetry (DSC) 204 of NETZSCH (Netzsch, Wittelsbacherstr, Germany) under a nitrogen atmosphere. Dielectric properties were measured with a GCSTD-A relative permittivity test instrument (Beijing Guance testing instrument Co., China) equipped by a heating apparatus. The ER effect of the ERFs was measured using a NXS-11B rotating viscometer from Chengdu instrument Co. (Chengdu, China).

\subsection{Synthesis}

Zinc tetraaminophthalocyanine was synthsized by the reduction of zinc tetranitrophthalocyanine with sodium sulfide according to previous report [5]. The precursor sulfonic acid-containing polysiloxane $\left(\mathrm{P}_{4}-\mathrm{SO}_{3} \mathrm{H}\right)$ were synthesized via poly(methylhydrogeno)siloxane, a sulfonic acid-containg monomer 4-(allyloxy)benzenesulfonic acid, a liquid crystal monomer cholesteryl 4-(allyloxy)benzoate, followed the methods described in our previous study, and the molar composition of cholesteryl mesogens derived from the monomer cholesteryl 4-(allyloxy)benzoate in the polymer systems were also reported in the work [6].

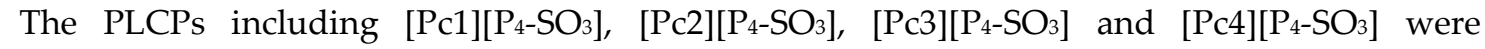
synthesized via ammonium salt reaction by use of $\mathrm{P}_{4}-\mathrm{SO}_{3} \mathrm{H}$ and zinc tetraaminophthalocyanine at the molar ratio (phthalocyanine: cholesteryl mesogens) of 9:1, 8:2 7:3 and 5:5, respectively. The synthetic diagram is shown in Figure 1a, and synthesis of $\left[\mathrm{Pc}_{4}\right]\left[\mathrm{P}_{4}-\mathrm{SO}_{3}\right]$ is given as an example. $\mathrm{P}_{4}-\mathrm{SO}_{3} \mathrm{H}(1.44$ $\mathrm{g}, 2.1 \mathrm{mmol}$ of cholesteryl mesogens) was dissolved in $50 \mathrm{~mL}$ of dry N-methyl-2-pyrrolidone by ultrasonic oscillation for $1 \mathrm{~h}$ under nitrogen condition. The solution was slowly dropped into zinc tetraaminophthalocyanine (1.38 g, $2.1 \mathrm{mmol}$, dissolved in $100 \mathrm{~mL}$ of N-methyl-2-pyrrolidone). The mixture was refluxed for $80 \mathrm{~min}$ in a $120 \mathrm{~W}$ microwave reactor. The solvent was distilled out under reduced pressure, and the residue was poured into $50 \mathrm{~mL}$ of acetone, and the solid was separated out. The solid was washed with successively with ethanol to give $2.52 \mathrm{~g}$ of $\left[\mathrm{Pc}_{4}\right]\left[\mathrm{P}_{4}-\mathrm{SO}_{3}\right]$. FTIR $(\mathrm{KBr}$, $\left.\mathrm{cm}^{-1}\right): 3651$ (m, O-H); 3348 (m, N-H); 3051, 2952, 2863, (vs, C-H); 1721 (s, C=O); 1601,1502 (s, C=C in phenyl); 1426 (s, C=N); 1466 (m, C=C in phthalocyanine); 1278 (s, C-O); 1178, 1121,1035 (vs, O=S); 1102, 1018 (vs, Si-O); 918, 850. Elem. Anal. Found: C, 63.15\%; H, 6.84\%; S, 1.92\%.

\subsection{Preparation of ER fluids}

The synthesized PLCPs were ground and sieved to give particles with maximum diameter 50 $\mu \mathrm{m}$. The ERFs were prepared by dispersing the particles (5 wt.\%) in silicone oil. The ERFs were stirred intensely by use of a homogenizator at $100 \mathrm{rpm}$ for $2.5 \mathrm{~h}$, and processed by ultrasonic oscillation for another $1.5 \mathrm{~h}$ to obtain better dispersion state. 


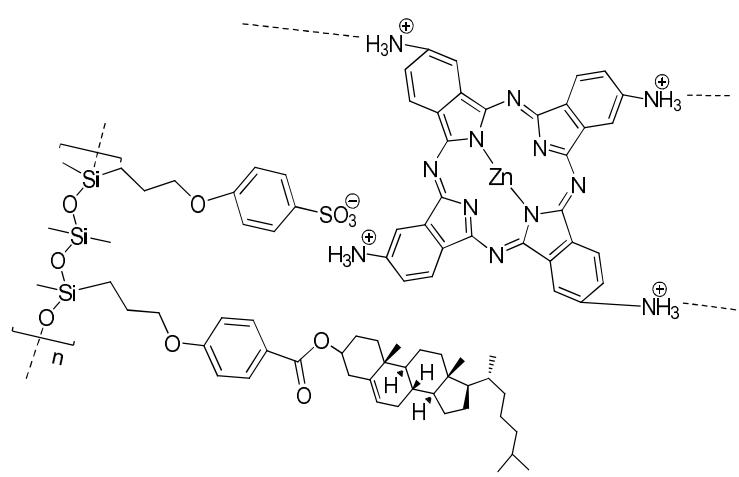

(a)

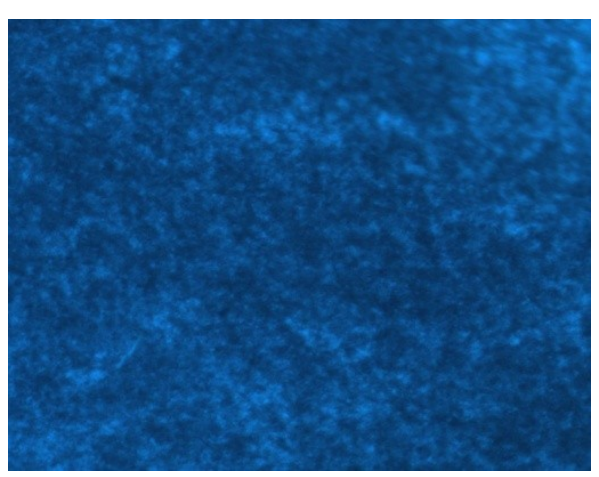

(b)

Figure 1. Synthetic diagram of the phthalocyanine-based liquid crystalline polysiloxanes (a), and columnar mesophase texture of $\left[\mathrm{P}_{2}\right]\left[\mathrm{P}_{4}-\mathrm{SO}_{3}\right]$ on heating to $92{ }^{\circ} \mathrm{C}(\mathbf{b})$.

\section{Results}

\subsection{Mesomorphic Property}

The liquid-crystalline behavior was investigated by optical textures observed under crosspolarized light and X-ray diffraction analysis as well as DSC measurement. The representative optical texture is shown in Figure 1b, and the phase transitions and XRD data is listed in Table 1.

Table 1. Phase transitions and XRD data of of phthalocyanine-based liquid crystalline polysiloxanes.

\begin{tabular}{|c|c|c|}
\hline Sample & Phase Transitions (Enthalpy Changes) ${ }^{1}$ & $\mathrm{XRD}^{2}$ \\
\hline$[\mathrm{Pc} 1]\left[\mathrm{P}_{4}-\mathrm{SO}_{3}\right]$ & G 58.1 Col 258.0 (2.94) I/I 226.1 (-2.01) Col 54.1 G & $36.9,21.3,18.1,13.8$ \\
\hline$\left[\mathrm{Pc}_{2}\right]\left[\mathrm{P}_{4}-\mathrm{SO}_{3}\right]$ & G 54.3 Col 256.1 (3.06) I/I $223.2(-2.07)$ Col 53.9 G & $37.2,21.4,18.3,14.0$ \\
\hline$[\mathrm{Pc} 3]\left[\mathrm{P}_{4}-\mathrm{SO}_{3}\right]$ & G 49.3 N 252.1 (2.87) I/I $221.2(-1.91)$ N 51.2 G & - \\
\hline$\left[\mathrm{Pc}_{4}\right]\left[\mathrm{P}_{4}-\mathrm{SO}_{3}\right]$ & G 46.3 N $249.1(2.46)$ I/I $219.2(-1.68)$ N 41.2 G & - \\
\hline
\end{tabular}

1 phase transition temperatures $\left({ }^{\circ} \mathrm{C}\right)$ and enthalpy changes $\left(\mathrm{J} \mathrm{g}^{-1}\right)$ in the second heating; $\mathrm{G}$, glassy; Col, columnar mesophase; $\mathrm{N}$, nematic; I, isotropic phase. ${ }^{2} d$-spacing in mesophase determined by XRD analysis.

\subsection{Dielectric Property and Electrorheological Effect}

The dielectric constant are investigated, and dielectric constant of the PLCPs as a function of temperature at a frequency of $1 \mathrm{kHz}$ is displayed in Figure 2a. The flow behaviors of shear stress as functions of shear rate for the PLCP-based ER fluids at constant conditions (the same electric field strength: $1.0 \mathrm{kV} \mathrm{mm}^{-1}$; temperature: $80^{\circ} \mathrm{C}$; concentration: $5 \mathrm{wt} . \%$ ) are shown in Figure $2 \mathrm{~b}$.

\section{Discussion}

As shown in Table 1, the mesophases of PLCPs change from nematic phase ([Pc3][ $\left.\mathrm{P}_{4}-\mathrm{SO}_{3}\right]$ and $\left.[\mathrm{Pc} 4]\left[\mathrm{P}_{4}-\mathrm{SO}_{3}\right]\right)$ to discotic hexagonal columnar mesophase ([Pc1][P4-SO $\left.\mathrm{P}_{3}\right]$ and $\left.[\mathrm{Pc} 2]\left[\mathrm{P}_{4}-\mathrm{SO}_{3}\right]\right)$ with increasing phthalocyanine component in the supermolecular systems. As shown in Figure 2a, it is observed that the dielectric constant increase with increasing phthalocyanine component in the PLCPs. When no external electric field was applied, the ER fluids show flow characteristics like the Newtonian fluid at different temperatures, but the non-Newtonian behavior with the application of an external electric field was confirmed. The shear stress of the PLCP-based ERFs strengthens firstly ([from $\left.\mathrm{Pc}_{4}\right]\left[\mathrm{P}_{4}-\mathrm{SO}_{3}\right]$ to $[\mathrm{Pc} 3]\left[\mathrm{P}_{4}-\mathrm{SO}_{3}\right]$ ) and then weakens with increase of phthalocyanine component

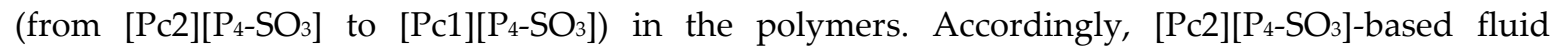
exhibited the strongest shear stress, as shown in Figure $2 \mathrm{~b}$. 


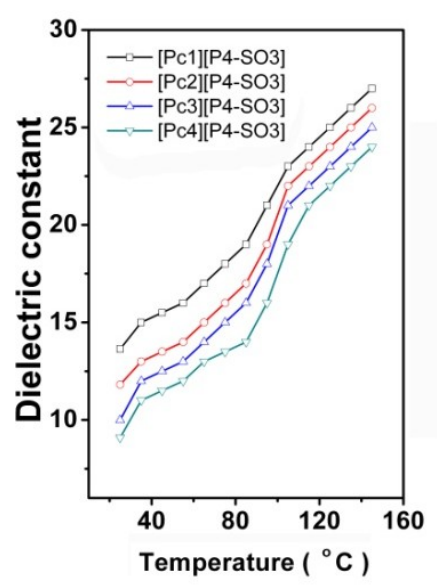

(a)

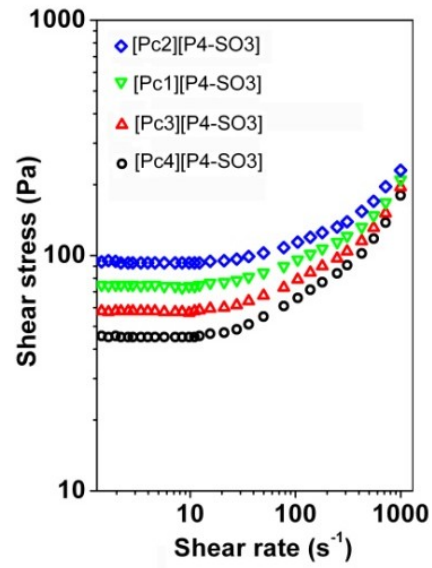

(b)

Figure 2. The dielectric constant as a function of temperature at a frequency of $1 \mathrm{kHz}(\mathbf{a})$, and shear stress as functions of shear rate for the PLCP-based ER fluids at constant conditions (the same electric field strength: $1.0 \mathrm{kV} \mathrm{mm}^{-1}$; temperature: $80^{\circ} \mathrm{C}$; concentration: $5 \mathrm{wt} . \%$ ).

\section{Conclusions}

In the present study, we prepared PLCPs using sulfonic acid-containg liquid crystalline polysiloxanes as polymer skeleton, and zinc tetraaminophthalocyanine as ionic crosslinkers. The PLCPs showed nematic and columnar mesophases. The dielectric constant increased with increasing phthalocyanine component in the polymer systems. The ER effect is strongest when the molar ratio of phthalocyanine and cholesteryl mesogens in the polymer is 8:2, suggesting the synergistic effect between semiconducting property of phthalocyanine and molecular orientation of the cholesteryl mesogens.

Author Contributions: F.M. designed the experiments, and wrote the paper; P.S. performed the experiments, and contributed reagents/materials/analysis tools; X.T. analyzed the data. All authors have read and agreed to the published version of the manuscript.

Acknowledgments: This work was supported by Key Laboratory of Green Energy and Environment Catalysis (Ningde Normal University), Fujian Province University [Project FJ-GEEC202002].

Conflicts of Interest: The authors declare no conflict of interest.

\section{References}

1. Vu, C.M.; Nguyen, V.H.; Bach, Q.V. Influences of electric field strength on rheological properties of electrorheological fluid based on hollow poly (o-phenylenediamine co o-toluidine) dispersed on silicone oil. J. Mol. Liq. 2020, 314,113762, doi:10.1016/j.molliq.2020.113762.

2. Trlica, J.; Saha, P.; Quadrat, O.; Stejskal, J. Electrorheological activity of polyphenylenediamine suspensions in silicone oil. Phys. A 2000, 283, 337-348, doi:10.1016/S0378-4371(00)00113-8.

3. Dong, Y.Z.; Seo, Y.; Choi, H.J. Recent development of electro-responsive smart electrorheological fluids. Soft Matt. 2019, 15, 3473-3486, doi:10.1039/C9SM00210C.

4. Bai, L.; Tang, X.; Gao, Y. Self-assembly of liquid crystalline polyethyleneimines bearing cholesteryl mesogens and ionic groups. New J. Chem. 2018, 42, 3236-3245, doi:10.1039/C7NJ04952H.

5. Meng, F.; Zhou, N.; Diao N.; Du, C. Discotic liquid crystal derived from zinc tetraaminophthalocyanine and perfluorooctanoic acid. Smart Mater. Struct. 2013, 22, 127002, doi:10.1088/0964-1726/22/12/127002.

6. Kong, S.; Song, Y.; Bai, L.; Tang, X.; Meng, F. Supramolecular complexes based on liquid-crystalline polysiloxanes and copper phthalocyanine. Polym. Inter. 2019, 68, 377-384, doi:10.1002/pi.5720.

Publisher's Note: MDPI stays neutral with regard to jurisdictional claims in published maps and institutional affiliations. 
(C) 2020 by the authors. Submitted for possible open access publication under the terms and conditions of the Creative Commons Attribution (CC BY) license (http://creativecommons.org/licenses/by/4.0/). 